\title{
Transient Dynamic Analysis of a Cantilever Rod with Axial Impulse Loading Using Finite Element Method
}

\author{
Sushmita Deka , Pallekonda Ramesh Babu and Maneswar Rahang \\ National Institute of Technology Meghalaya, India
}

\begin{abstract}
The behavior of bodies subjected to impulse loading is of prime importance in the study of forces that occur in impulse facilities. Before performing the actual tests, theoretical and numerical simulations are carried out to obtain the response of bodies subjected to impulse loading. The simplest model for this study can be considered as a rod of circular cross section fixed at one end and free at other end. When a transient impulse load is applied on a body, vibrations occur in the body for the brief period of time. In this paper, the effect of a half sine impulse force applied on a cantilever rod in the axial direction has been discussed. The displacements at the tip of the rod were obtained based on two theories, the basic vibration formulae and FEM analysis. Simulations were performed using ANSYS and compared with the displacements obtained from the two theoretical methods.
\end{abstract}

\section{Introduction}

Impulse facilities like wind tunnels and shock tunnels are used for testing of various scaled aerodynamic models to find the forces and moments which it undergoes when subjected to various types of loads. These facilities are of short test duration in the order of milliseconds. The theoretical and numerical analysis to find forces and moments are generally carried out before actual testing in shock tunnels and wind tunnels. These analysis are usually performed by Computational Fluid Dynamics (CFD) and Finite Element (FE) simulations. However, the vibrations occurred in the body due to the applied forces can also be studied to know the effect of the forces on the body. The vibrations result in deformation of the body, and the forces can be predicted from various techniques by analyzing the deformation occurring on the body as a result of the applied force. Some of the researchers have worked on determining the response of the system subjected to various kinds of loads. Jia-shi et al. [1] studied the longitudinal forced vibration of a rod with non-linear boundary conditions having a concentrated mass at one end and supported by a spring on the other end. Wei [2] reviewed the vibration analysis of strings, rods, beams, membranes and thin plates using the discrete singular convolution algorithm. Temel and Calim [3] studied the forced vibration of cylindrical helical cantilever rod subjected to impulse loading in the Lapalce domain using the complementary functions method. The Timoshenko beam theory was used to derive the governing equations for cylindrical helical rods. The displacements and moments obtained were compared with those obtained from ANSYS simulations. Arani et al. [4] calculated the natural frequencies and mode shapes of rods with variable cross section considering various boundary conditions like clamped-clamped, clamped-free, free-free and freeclamped. They used a wave propagation method to find the natural frequency and mode shapes and compared it with the analytical method. A higher accuracy was obtained from the wave propagation method than the analytical method. Shokrollohi and Nejad [5] studied the longitudinal free vibration of non-uniform clampedclamped and clamped-free rods to find the natural frequency by using discrete singular convolution. Kruti [6] studied the free longitudinal vibrations of a straight rod fixed at one end to find the natural frequencies of the rod. The rod was considered with uniform properties throughout the length of the rod and the method of force integration was used. Mei [7] studied the vibrations in uniform and stepped rods using four rod theories, the elementary theory, the Love theory, the MindlinHerrman theory and the three mode theory in which the motion of the rod is described by the propagation and reflection of waves through the rod. Shali et al. [8] studied the vibration of non-uniform rods having clamped-clamped and clamped-free ends to find the natural frequency using differential transform method by solving ordinary and partial differential equations. Collini et al. [9] studied the vibration analysis of tie rods having elastic bed type boundary conditions and 
compared with a model having both end fixed boundary conditions. The natural frequency of the tie rod was found from which the axial loads on the tie rod was determined. The vibrations on a rod due to an applied force were also studied using finite element analysis by researchers. Mottershead [10] studied the vibrations of a helical rod using finite element analysis to find the natural frequency of the helical rod. The frequency obtained was compared with those of a helical spring. Karami et al. [11] studied the free vibrations of spatial rods fixed at one end using finite element analysis. Adhikari et al. [12] studied the free and forced axial vibrations of rods with non-local elasticity using finite element analysis.

Although a considerable amount of work has been carried out on the study of vibration of cantilever rods, very limited work has been reported in literature in comparing the response using analytical techniques such as vibration formulae and the finite element method. In this paper, a solid rod of circular cross section made of aluminium has been considered for the analysis. Axial half sine impulse force has been considered to apply on the model to find the displacement at the tip of the rod due to the force. The analysis is performed using vibration formulae and FEM analysis along with simulations in ANSYS. The displacements obtained from the three techniques were compared with each other. The schematic diagram of the cantilever rod and a half sine impulse force is shown in Figure 1(a) and Figure 1(b).

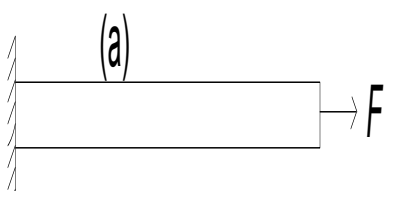

Figure 1: (a) Schematic diagram of a cantilever rod

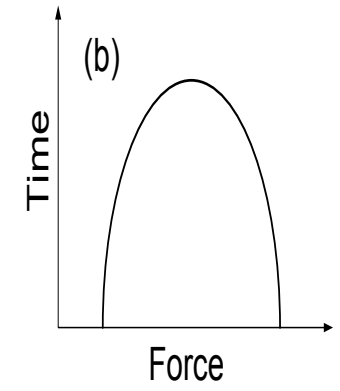

(b) Half sine impulse force

\section{Formulation}

To find the displacement at the tip of the cantilever rod subjected to impulse load, the vibration theory and the FEM theory has been used. The displacement at the tip of the cantilever rod is measured for transient loading. The transient load is considered to follow a harmonic behavior with very short time duration in the order of milliseconds. The force is acting at the end of the rod in the axial direction i.e. no deformation occurs in the $y$ and $\mathrm{z}$ direction. The rod cross section is considered to be circular and the displacement at the tip of the rod is found for various lengths and loads on the rod. The displacement at the tip of the rod is also found for different values of time for transient loading. Let the length of a cantilever rod be $L$ on which an axial transient load $f=F \sin \omega t$ is acting at the end of the rod, where $F$ is the amplitude of the load, $\omega$ is the frequency of the load and $t$ is the time for which the load is acting. Let the stiffness of the rod be $\mathrm{k}$ and the displacement at the tip of the rod be denoted by $u$. For a constant force $F$,

Force $(F)=$ Stiffness $(k) \times$ displacement $(u)$

$$
\text { i.e. } \quad u=F / k
$$

Here $E$ is the Young's modulus, $A$ is the cross section area of the rod and $l$ is the length of each element of the rod. The generalized stiffness and force matrices considering 3 elements of the rod are represented as given below using FEM,

$$
\begin{gathered}
k=\frac{E A}{l}\left[\begin{array}{cccc}
1 & -1 & 0 & 0 \\
-1 & 2 & -1 & 0 \\
0 & -1 & 2 & -1 \\
0 & 0 & -1 & 1
\end{array}\right] \\
F=\left[\begin{array}{l}
F_{1} \\
F_{2} \\
F_{3} \\
F_{4}
\end{array}\right]=\left[\begin{array}{l}
0 \\
0 \\
0 \\
F
\end{array}\right]
\end{gathered}
$$

Displacement at each node,

$$
\left[\begin{array}{l}
u_{1} \\
u_{2} \\
u_{3} \\
u_{4}
\end{array}\right]=\frac{E A}{l}\left[\begin{array}{cccc}
1 & -1 & 0 & 0 \\
-1 & 2 & -1 & 0 \\
0 & -1 & 2 & -1 \\
0 & 0 & -1 & 1
\end{array}\right]^{-1}\left[\begin{array}{l}
0 \\
0 \\
0 \\
F
\end{array}\right]
$$

The force considered in the present condition is transient impulse force in the form, $f=F \sin \omega t$.

The displacements with respect to time at any node denoted as $U$ and the expression for the same is given by

$$
U=k^{-1} F \sin \omega t
$$

where $t$ varies from zero to the total time.

According to vibration theory, the equation of motion of a rod in axial direction is given by

$$
\frac{\partial}{\partial x}\left(E A \frac{\partial u}{\partial x}\right)=\rho A \frac{\partial^{2} u}{\partial t^{2}}
$$

where $\rho$ is the density of the material of the rod.

If the property of the rod do not change with length, then

$$
\frac{\partial^{2} u}{\partial t^{2}}=c^{2} \frac{\partial^{2} u}{\partial x^{2}}
$$

where $c^{2}=\frac{E}{\rho}$ is the speed of sound in the rod. The equation (7) is the one dimensional wave equation which describes the propagation of electromagnetic waves in a homogenous medium. The boundary conditions of a cantilever rod fixed at one end are

$$
u(0, t)=0, \frac{\partial u}{\partial x}(l, t)=0
$$


Equation (7) can be solved as an eigen value problem for vibration of a rod using method of separation of variables i.e.

$$
u(x, t)=U(x) T(t)
$$

which gives the following equation

$$
\frac{1}{T(t)} \frac{d^{2} T}{d t^{2}}=c^{2} \frac{1}{U(x)} \frac{d^{2} U}{d x^{2}}
$$

Let each of the terms in equation (9) be equal to a constant $-\omega^{2}$. Thus

$$
\frac{1}{T(t)} \frac{d^{2} T}{d t^{2}}=-\omega^{2}
$$

And

$$
c^{2} \frac{1}{U(x)} \frac{d^{2} U}{d x^{2}}=-\omega^{2}
$$

On solving the equations (10) and (11) and applying the boundary conditions, the natural frequency $\left(\omega_{n}\right)$ of the cantilever rod is given the following formula [13],

$$
\omega_{n}=\frac{\pi \sqrt{E / \rho}}{2 L}
$$

For transient impulse force, $f=F \sin \omega t$, displacements with respect to time during the pulse duration at any node is given by [14],

$$
U=\frac{F / k}{1-\left(\frac{\omega}{\omega_{n}}\right)^{2}}\left[\sin (\omega t)-\left(\frac{\omega}{\omega_{n}}\right) \sin \left(\omega_{n} t\right)\right]
$$

where $t$ varies from zero to the total time.

After the pulse, the displacement with respect to time is given by [14],

$$
U=\frac{F / k}{1-\left(\frac{\omega}{\omega_{n}}\right)^{2}}\left(\frac{\omega}{\omega_{n}}\right)\left[\sin \left(\omega_{n} t\right)-\sin \left(\omega_{n}\left(t-t^{\prime}\right)\right)\right]
$$

where $t^{\prime}$ is the impulse duration.

\section{Results and discussion}

\subsection{Effect of the length of the rod on the displacement at the tip}

The half sine impulse force $f=F \sin \omega t$, having an amplitude of $10 \mathrm{~N}$ has been applied on the aluminum rod with $0.01 \mathrm{~m}$ diameter for a time of $1 \mathrm{~ms}$. The displacement at the tip of the cantilever rod is plotted for three different lengths i.e. $0.2 \mathrm{~m}, 0.5 \mathrm{~m}$ and $0.8 \mathrm{~m}$. The displacement history obtained using the analytical FEM and vibration theory for the applied forces are shown in Figure 2(a) and Figure 2(b).

The displacement at the free end of the rod increases with increase in the length. Since the stiffness of a body is inversely proportional to the length, hence the displacement at the end of the rod increases with increasing length.
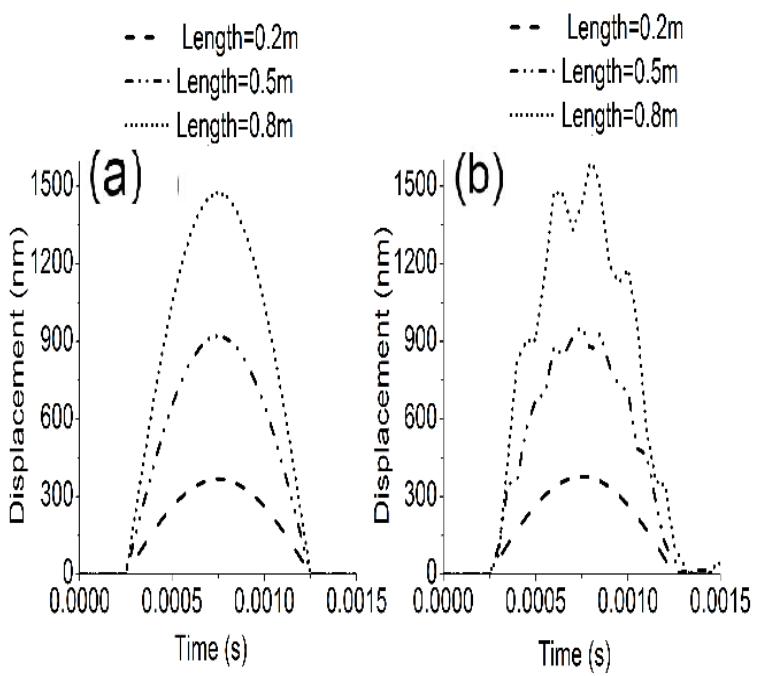

Figure 2: (a) Displacement history obtained from analytical FEM method of the rods with different lengths.

(b): Displacement history obtained from vibration analysis of the rods with different lengths.

Numerical simulations were performed in ANSYS using finite element method for different lengths of the rod and is compared with the displacements obtained from analytical FEM and vibration analysis. The parameters were kept the same as those used for the two techniques. One end of the rod was fixed while the other end was free and the displacement was considered only in the axial direction. Hexahedral elements were used for the simulation and the number of element were 560. The displacements obtained from the ANSYS simulations for three given lengths are given in Figure 3. The obtained displacements agree well with the displacements obtained from the theoretical techniques. The displacements obtained from FEM, vibration and ANSYS simulations for $0.2 \mathrm{~m}$ length of rod are compared in Figure 4.

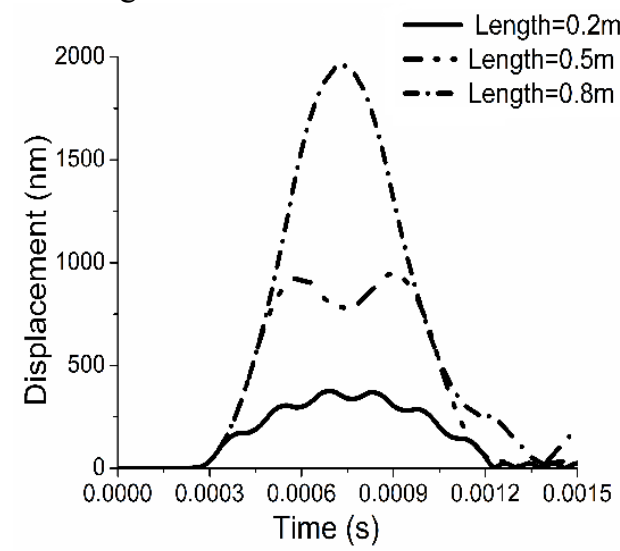

Figure 3: Variation of displacement with time at three different lengths obtained using ANSYS 


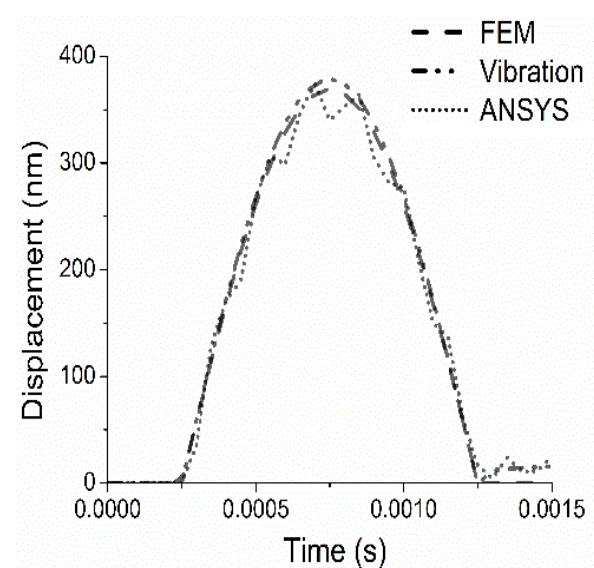

Figure 4: Variation of displacement with time for $0.2 \mathrm{~m}$ length of rod obtained using FEM, vibration and ANSYS

\subsection{Displacement at the tip of the cantilever rod on varying the load}

The displacement history obtained at the tip of the cantilever rod was plotted for three different amplitude of loads applied at free end of the rod, i.e. $10 \mathrm{~N}, 20 \mathrm{~N}$ and $30 \mathrm{~N}$. The force is considered to act harmonically following the equation $f=F \sin \omega t$ for a time of $1 \mathrm{~ms}$. The other parameters were kept the same for three cases. The graphs obtained using the FEM analysis and vibration theory are shown in Figure 5(a) and Figure $5(b)$.

The displacement at the free end of the rod increases with increase in amplitude of load. The displacements obtained from the FEM analysis and vibration analysis are found to agree well with each other.
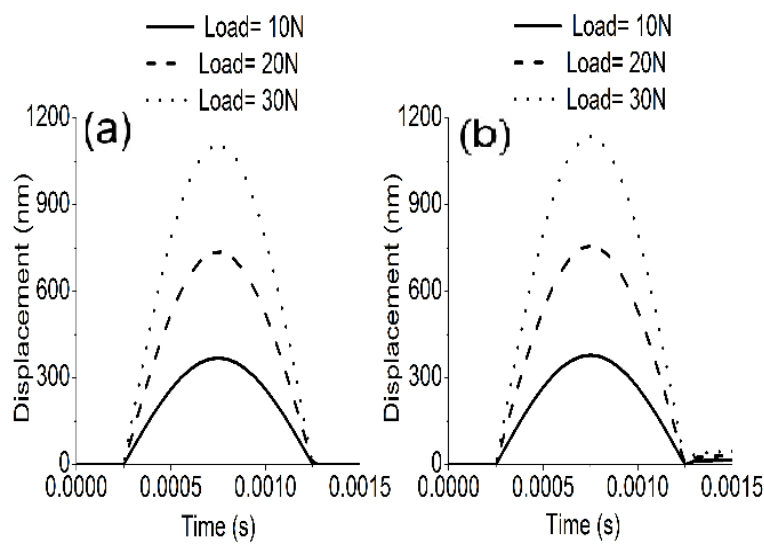

Figure 5 : (a)Variation of displacement with time at three different amplitude of loads obtained from FEM analysis

(b):Variation

of displacement with time at three different amplitude of loads obtained from vibration analysis

Using the same ANSYS model as used earlier, the simulations were performed to find the displacement at the free end of the rod for three different amplitude of loads and the displacements were found to agree well with the theoretical results. The displacements obtained from the simulation are shown in Figure 6.

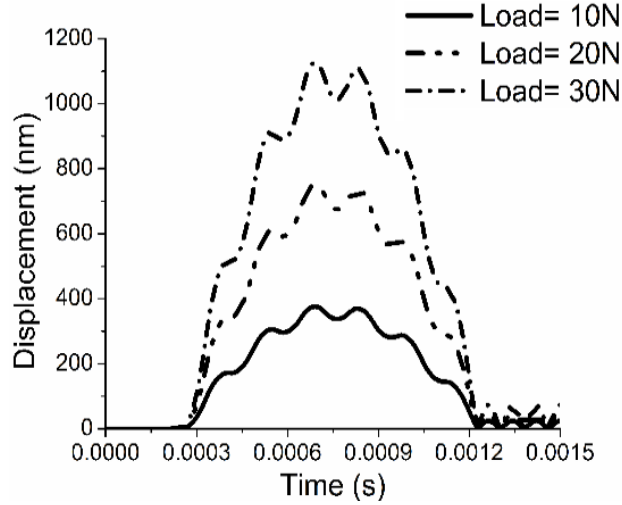

Figure 6: Variation of displacement with time at three different loads obtained using ANSYS

\subsection{Displacement at the tip of the cantilever rod on varying the total time of impulse}

The total time for which the harmonic load is applied is varied for three different times- $1 \mathrm{~ms}, 2 \mathrm{~ms}$ and $3 \mathrm{~ms}$. The effect of the varying impulse time on the displacement at the free end of the cantilever rod is analyzed keeping other parameters like length of the rod and amplitude of the load as constant for the three cases. The length of the rod is considered as $0.2 \mathrm{~m}$ and amplitude of the load applied is considered as $10 \mathrm{~N}$. The graphs obtained using the FEM analysis and vibration theory are shown in Figure 7. It is found that the maximum displacement at the tip of the rod remains same for all the three duration of impulses for both the theoretical methods. Both the theoretical methods to find the displacement are found to agree well with each other.
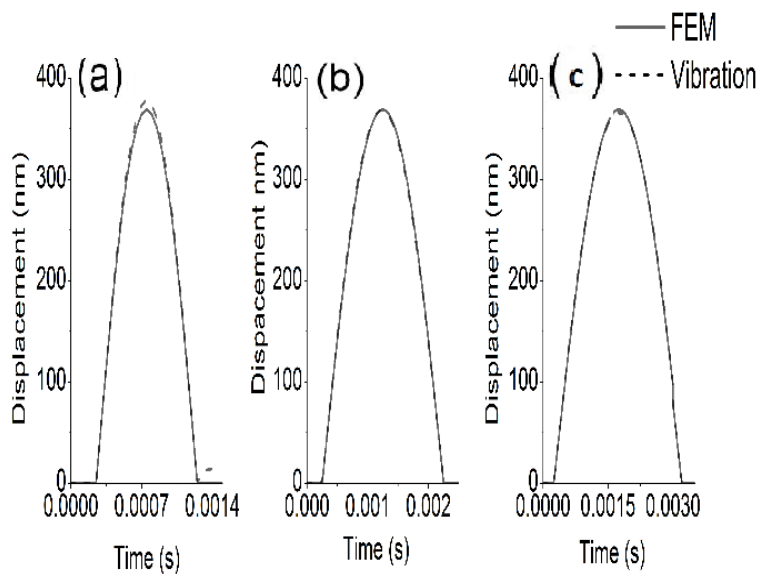

Figure 7: Variation of displacement with time at three different impulse durations obtained from FEM analysis and vibration analysis

The same ANSYS model is again used to perform simulations to analyze the effect of different impulse duration on the displacement at the tip of the cantilever rod. The displacements obtained agreed well with those obtained from theoretical analysis. The maximum displacement is almost same for all the three time durations. The displacements obtained from the ANSYS simulations are shown in Figure 8. 


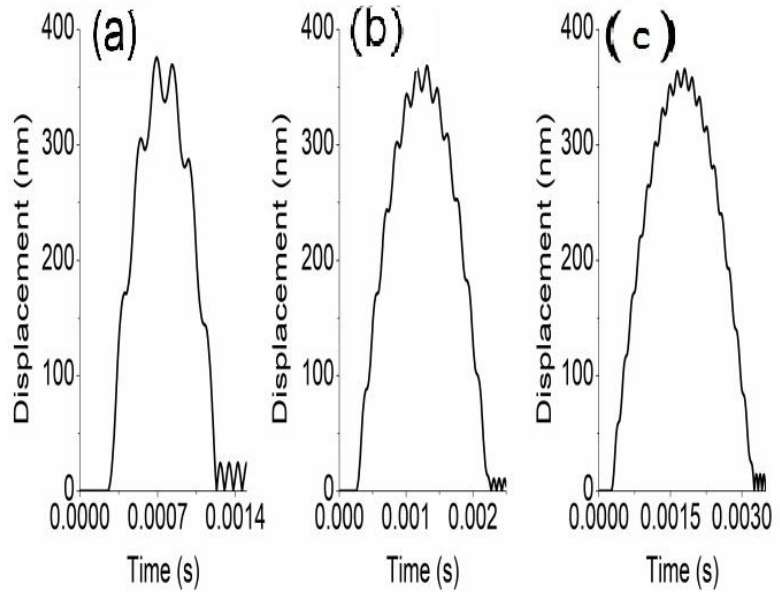

Figure 8: Variation of displacement with time at three different impulse durations obtained from ANSYS

\section{Conclusion}

In the present study, a half sine impulse force is applied on a cantilever rod made of aluminium and the displacement is measured at the tip of the rod for different lengths, different amplitudes of loads and different impulse duration. The analytical FEM theory and the vibration theory was used to measure the displacement at the tip of the rod. The displacements obtained from both the methods are compared with each other and good agreement has been found between the two theories. The displacements are also compared with the displacements obtained from ANSYS simulations and they were found to agree well with those obtained from theoretical analysis. However, the displacements obtained from FEM and vibration theory were found to be smoother compared to that obtained from the ANSYS simulations.

\section{References}

1. T. Jia-shi, L. Li and H. Quan-zhong, Vibration analysis of a rod with complex boundary conditions, Appl. Math. Mech. 9(1988), 837847

2. G. W. Wei, Vibration analysis by discrete singular convolution, J sound Vib. 244(2001), 535-553

3. B. Temel and F. F. Calim, Forced vibration of cylindrical helical rods subjected to impulsive loads. J. Appl. Mech. 70(2003), 281-291.
4. M. K. Arani, N. R. Saleh and M. N. Bahrami, A Modified Wave Approach for the Calculation of Natural Frequencies and Mode Shapes of the Rods with Variable Cross Section, App. Mech. Mat. 110(2012), 2537 2547

5. M. Shokrollahi, and A. Z. B. Nejad, Numerical analysis of free longitudinal vibration of nonuniform rods: Discrete singular convolution approach, J ENG MECH ASCE. 140 (2014), 06014007

6. Y. Krutii, Analysis of longitudinal oscillations for systems with continuous variable parameters using force integration method, Tehnicki glasnik, 9(2015), 420-425

7. C. Mei, Comparison of the four rod theories of longitudinally vibrating rods, J. Vib. Control. 21(2015), 1639-1656

8. S. Shali, S. R. Nagaraja, and P. Jafarali, Vibration of non-uniform rod using Differential Transform Method, Mater. Sci. Eng. 225(2017), 012027

9. L. Collini, R. Garziera, and K. Riabova, Vibration Analysis for Monitoring of Ancient Tie-Rods, SHOCK VIB. 2017

10. J. E. Mottershead, Finite elements for dynamical analysis of helical rods, Int. J. Mech. Sc. 22(1980), 267-283.

11. G. Karami, M. Farshad, and M. Yazdchi, Free vibrations of spatial rods-A finite-element analysis, Int J Numer Method Biomed Eng. 6(1990), 417-428.

12. S. Adhikari, T. Murmu, and M. A. McCarthy, Dynamic finite element analysis of axially vibrating nonlocal rods, FINITE ELEM ANAL DES. 63(2013), 42-50.

13. W.T. Thomsn, M.D. Dahleh, C. Padmanabhan, Theory of Vibration with Applications. $5^{\text {th }}$ edn, Pearson Education (1998)

14. L. Meironvtch, Fundamentals of Vibration, Waveland Press (2010) 